\title{
Clinical Trial Results with the MED-EL Fine Structure Processing Coding Strategy in Experienced Cochlear Implant Users
}

\author{
Joachim Müller ${ }^{a}$ Stefan Brill ${ }^{b}$ Rudolf Hagen ${ }^{b} \quad$ Alexander Moeltner $^{b}$ \\ Steffi-Johanna Brockmeier ${ }^{c, h}$ Thomas Stark ${ }^{c, d}$ Silke Helbig ${ }^{e} \quad J_{a n}$ Maurer $^{f}$ \\ Thomas Zahnert ${ }^{\mathrm{g}}$ Clemens Zierhofer ${ }^{\mathrm{i}}$ Peter Nopp ${ }^{\mathrm{j}} \quad$ llona Anderson $^{\mathrm{j}}$ \\ a Department of Otorhinolaryngology, Klinik und Poliklinik für Hals-Nasen-Ohren-Heilkunde, Ludwig-Maximilians- \\ Universität München, Munich, ${ }^{b}$ Department of Otorhinolaryngology, Plastic, Aesthetic and Reconstructive Head \\ and Neck Surgery, University of Würzburg, Würzburg, ' ${ }^{C H a l s-}$, Nasen-, Ohrenklinik und Poliklinikum rechts der \\ Isar, Technische Universität München, Munich, dSt. Elisabeth Hospital, HNO-Klinik der Ruhr Universität, Bochum,

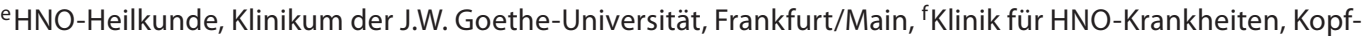 \\ Hals-Schädelbasis-Chirurgie, plastische Operationen und Centrum für Hören und Kommunikation Katholisches

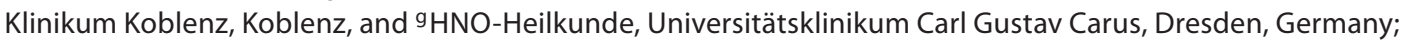 \\ h Department of Pedaudiology, University of Basel, Basel, Switzerland; ' $C$ Christian Doppler Laboratory for Active \\ Implantable Systems, Institute of Applied Physics, University of Innsbruck, and j MED-EL GmbH, Innsbruck, Austria
}

\section{Key Words}

Fine structure processing $\cdot$ Cochlear implant $\cdot$ Coding

strategy $\cdot$ Speech perception $\cdot$ Music $\cdot$ Pitch $\cdot$ CIS+ $\cdot$ OPUS

\begin{abstract}
Objectives: To assess the subjective and objective performance of the new fine structure processing strategy (FSP) compared to the previous generation coding strategies CIS+ and HDCIS. Methods: Forty-six adults with a minimum of 6 months of cochlear implant experience were included. CIS+, HDCIS and FSP were compared in speech perception tests in noise, pitch scaling and questionnaires. The randomized tests were performed acutely (interval 1) and again after 3 months of FSP experience (interval 3). The subjective evaluation included questionnaire 1 at intervals 1 and 3 , and questionnaire 2 at interval 2, 1 month after interval 1. Results: Comparison between FSP and CIS+ showed that FSP performed at least as well as CIS+ in all speech perception tests, and outperformed CIS+ in vowel and monosyllabic word dis-
\end{abstract}

\section{KARGER}

Fax +4161306 1234

E-Mail karger@karger.ch

www.karger.com
(C) 2012 S. Karger AG, Basel

0301-1569/12/0744-0185\$38.00/0

Accessible online at:

www.karger.com/orl crimination. Comparison between FSP and HDCIS showed that both performed equally well in all speech perception tests. Pitch scaling showed that FSP performed at least as well as HDCIS. With FSP, sound quality was at least as good and often better than with HDCIS. Conclusions: Results indicate that FSP performs better than CIS+ in vowel and monosyllabic word understanding. Subjective evaluation demonstrates strong user preferences for FSP when listening to speech and music.

Copyright $\odot 2012$ S. Karger AG, Basel

\section{Introduction}

A sound stimulus can be decomposed into amplitude, frequency and phase signals using the Hilbert transform [1]. Thus, we can regard a signal as being a constant amplitude, fine structure carrier, which is amplitude-modulated by an envelope signal. In speech and other acoustic signals, the fine structure varies continuously and carries 
important information such as pitch and timbre. In normal hearing, the neural response to low frequencies reflects both the envelope and the fine structure information of the signal, whereas in the neural response to higher frequencies beyond around $5 \mathrm{kHz}$, the envelope is mainly represented [2].

In normal-hearing subjects, when presenting signals that are limited to a number of independent channels as used in cochlear implants (CIs) today, the primary information carrier for (western) speech is the envelope, whereas for music it is the fine structure [3,4]. Smith et al. [3] also found that interaural time delays in the fine structure are more strongly determining perceived stimulus site than interaural time delays in the envelope, although the speech signal coded in the envelope still dominates perception.

All continuous-interleaved-sampling (CIS) and n-of$\mathrm{m}$-based coding strategies that have been in use in CIs during the last 15-20 years rely mainly on envelope information [5]. In general, users of these coding strategies show good to very good speech perception in quiet, moderate speech perception in noise and poor to moderate music appreciation [6]. Specifically, the transmission of tonal speech information, such as prosodic contour or speaker gender, as well as music perception and appreciation is poor in CI users compared to normal-hearing listeners [7-9].

Thus, the quintessential performance characteristics of these coding strategies are in agreement with the results found by Smith et al. [3]. The observation that envelope information alone is suitable for supporting very good speech understanding but only moderate music appreciation suggests that these strategies do not provide sufficient fine structure information required for a better performance in music perception.

This notion is supported by results from users of electric-acoustic stimulation (EAS), an approach to cochlear implantation first described by von Ilberg et al. [10] that has become widely accepted. In EAS, individuals with a ski slope type hearing loss are treated by combining a CI and a hearing aid in the same ear. The CI electrode is shorter than usual and covers the cochlea only partially and thus provides the mid- to high-frequency range. Low frequencies are amplified by the hearing aid, given the residual hearing could be preserved during CI surgery. In individuals with little to no low-frequency hearing loss, amplification may not be required at all. In contrast to regular CI users, EAS recipients enjoy acoustic hearing in the low frequencies, and thus have access to both envelope and fine structure information.
Results reported in users of EAS demonstrate improved speech perception in noise and improved music appreciation [11]. Therefore, EAS users perform better in exactly those conditions where standard CI users experience the most difficulty. These results are in line with Smith et al. [3], in that they further demonstrate how essential the fine structure component is for sound perception. The major difference between EAS and regular CI with complete cochlear coverage is the presence of fine structure information in the low-frequency region. Therefore, the results from EAS also suggest that improved temporal coding in the low frequencies could enhance CI performance.

To improve the transmittance of fine structure information, the fine structure processing (FSP) coding strategy was developed by MED-EL (Innsbruck, Austria). FSP is intended to better enable users to perceive pitch variations and timing details of sound. The FSP as well as the HDCIS (high-definition CIS) coding strategies are available in the MED-EL OPUS 1 and OPUS 2 processors.

This paper reports and discusses the results of a multicentre clinical trial investigating the effect of the FSP strategy on auditory perception in CI users compared to the HDCIS and the CIS+ strategies available in the TEMPO+ speech processor, the predecessor of the OPUS audio processors. The objective of this study was to test if the FSP strategy was superior or non-inferior to the HDCIS strategy and if the FSP and HDCIS coding strategies were superior or non-inferior to the CIS+ strategy.

\section{Methods}

\section{Subjects}

Forty-six adult subjects, who had received a PULSARCI ${ }^{100} \mathrm{CI}$ unilaterally, were enrolled into the MED-EL fine structure clinical trial ( 24 female, 22 male). They were recruited from 13 centres across Germany. All subjects were postlingually deafened, except for 1 subject who had an onset of progressive hearing loss at 2 years of age. However, we considered this subject to fulfil the inclusion criteria of postlingual deafness since the progressive hearing loss allowed the use of natural hearing during the early stages of speech and language development. Subjects had a minimum of 6 months of experience with the CIS + coding strategy, and had to score better than $40 \%$ on Freiburg monosyllables to be able to complete the speech perception tests included in this study design. Aetiologies of hearing loss were: progressive (4), otosclerosis (2), trauma (1), sudden hearing loss (1), noise trauma (1), NF2 (1), Usher's syndrome (1), viral infection (1), Ménière's disease (1), progressive hearing loss and flu infection (1), cholesteatoma (1), middle ear infection (1), genetic (suspected, 1) and other (1). In 28 subjects, the aetiology was unknown. The subjects' mean age at hearing loss was 46 years (range: $2-71$ ). Their mean age at implan- 
tation was 54 years (range: $20-76$ ) and their mean age at testing was 56 years (range: 20-77) with a mean CI experience of 1.4 years (range: 7-31 months).

Subjects were required to have at least 10 active electrodes as defined at the most recent fitting before the beginning of the study. Five subjects had 10, 11 subjects had 11, and 30 subjects had 12 electrodes activated. Subjects were also required to have an overall stimulation rate $\geq 15,600$ pps for CIS+ with the TEMPO+ speech processor. Most comfortable loudness levels of active electrodes had to be below compliance level.

\section{Devices and Strategies}

Subjects came into the study using their clinically fitted TEMPO+ speech processor and were switched over to the OPUS 1 audio processor. The OPUS 1 allows either the slightly modified CIS+ speech coding (HDCIS) or the new FSP coding strategy. HDCIS operates equivalently to the CIS+ strategy in the TEMPO+ speech processor [12]. Just like CIS+, HDCIS is an envelope-based strategy, where the envelope of the sound signal in a certain frequency range is sampled, using a constant-rate pulse train that bears no relationship to the temporal structure of the sound signal. Thus, frequency is presented only using the place cue, in other words, presented only via the location of the stimulated electrode contact in the cochlea. Beyond the usual envelope modulations in CIS-like strategies that mainly transmit fundamental frequency, the temporal fine structure of the sound signal is not represented.

In contrast to fixed-rate envelope-based coding strategies like HDCIS where the timing of stimulation is not an information carrier, FSP works in both time and place. The timing of stimulation is used to code the temporal structure of the sound signal particularly in the low- to mid-frequency range. This is achieved by using channel-specific sampling sequences (CSSS) [13]. A CSSS is a series of stimulation pulses that is started at each positive-going zero-crossing in a channel's band-pass filter output. In FSP, the length of these sequences is related to the band-pass filter's upper corner frequency. Thus, the instantaneous repetition rate of these sequences equals the instantaneous fine structure frequency of the signal in the respective frequency range. In the FSP strategy, CSSS is typically used on the lower (i.e. apical) 2-3 channels, which means that depending on the band-pass filter arrangement, the temporal fine structure is coded using CSSS for frequencies up to $300-500 \mathrm{~Hz}$ and the lower cut-off frequency is reduced from 250 $\mathrm{Hz}$ used for the CIS+ and HDCIS strategies [14] to between 70 and $100 \mathrm{~Hz}[14,15]$ for FSP. For the current study, the clinical settings in the fitting software for the lower cut-off frequency were used: i.e. $100 \mathrm{~Hz}$ for FSP and $250 \mathrm{~Hz}$ for CIS+ and HDCIS.

On the remaining channels, tonotopic frequency coding is achieved using 'virtual channels', which are pitch percepts that are intermediate to the pitch percepts created by stimulating single electrodes in isolation $[16,17]$. Just like HDCIS, FSP uses bandpass filters with a bell-shaped frequency response [18]. This allows a smooth transition of stimulation from one electrode to the adjacent apical or basal electrode as frequency decreases or increases. As an example, stimulation amplitude on the more apical electrode will decrease and stimulation amplitude on the more basal electrode will increase, as input frequency increases. It was found in a study by Nobbe et al. [18] that this filter design in conjunction with sequential stimulation is as efficient in creating intermediate pitches (and thus 'virtual channels') as simultaneous stimulation.
Table 1. Overview of tests conducted for each coding strategy

\begin{tabular}{|c|c|c|c|}
\hline & $\begin{array}{l}\text { CIS+ } \\
(\mathrm{TEMPO}+)\end{array}$ & $\begin{array}{l}\text { HDCIS } \\
\text { (OPUS 1) }\end{array}$ & $\begin{array}{l}\text { FSP } \\
\text { (OPUS 1) }\end{array}$ \\
\hline Speech tests (intervals 1 and 3 ) & + & + & + \\
\hline Questionnaire 1 (intervals 1 and 3) & & + & + \\
\hline Questionnaire 2 (interval 2) & + & & + \\
\hline Pitch scaling (intervals 1 and 3 ) & & + & + \\
\hline
\end{tabular}

\section{Study Design}

All subjects were fitted with the OPUS 1 behind-the-ear audio processor at the acute test interval (test interval 1). During test interval 1, they were assessed using the CIS+ (TEMPO+), the HDCIS (OPUS 1) and the FSP (OPUS 1) speech coding strategies on sentences in noise, monosyllables in noise, and vowels in noise. Subjects also completed a questionnaire (questionnaire 1). In this questionnaire, the subjects compared the HDCIS and FSP strategies on the OPUS 1, toggling between the two programmes to make a comparative judgement. Finally, subjects completed a pitch scaling test, comparing the HDCIS and FSP strategies on the OPUS 1.

Subjects then wore the OPUS 1 audio processor using FSP. After 1 month (test interval 2), they completed a postal questionnaire (questionnaire 2) comparing listening experience with the OPUS 1 (FSP) to that with the TEMPO+ (CIS+). After 3 months of OPUS 1 experience with FSP (test interval 3), which allowed sufficient time for the subjects to become accustomed to the new coding strategy, subjects returned to the clinic to be reassessed using the same test protocol as for test interval 1 (see table 1 for an overview of tests, coding strategies and test interval combinations).

The study received all necessary ethics board approvals and subjects signed an informed consent before their enrolment in the study. Subjects were reimbursed for travel expenses and received a compensation payment after finishing the study. If a subject decided to prematurely terminate the study, then he/she received a proportionate compensation payment.

\section{Questionnaires}

In questionnaire 1, subjects were asked to compare their hearing impression of music and speech with the FSP and HDCIS strategies, which were tested in a randomized order. The stimuli were presented via headphones (Sennheiser HD570). Subjects listened to two pieces of music - a well-known German nursery rhyme ('Hänschen klein', same melody as 'Little John') and Mozart's 'Little Night Music' - as well as to a male-female dialogue in quiet and in noise. Twenty-four questions were competitive on a 5-step scale; these were included to test the hypothesis of FSP being equal to or better than the HDCIS strategy. For the 5-step scale questions, categories 3-5 were considered to be 'equal to' or 'better than'. The analyses of these questions are shown on a percentage scale demonstrating the proportion of subjects scoring in categories 3-5 in order to test the hypothesis. Questionnaire 1 furthermore included 6 questions on timbre of sound and pitch of the voice. These questions were not of a competitive nature; 
their only purpose was to obtain an additional idea of the quality of the different strategies, i.e. they were not used to measure the efficacy of strategies.

In questionnaire 2 , which was a postal questionnaire, subjects were asked to retrospectively compare their hearing with the FSP strategy (OPUS 1) to the hearing they had when using the CIS+ strategy (TEMPO+). The rationale behind administering questionnaire 2 after 1 month was based on the assumption that subjects could still remember their experience with the TEMPO+ speech processor, i.e. with the CIS+ strategy, and could therefore retrospectively answer competitive questions between FSP and CIS+. It was furthermore expected that subjects would have had sufficient time to adjust to the new coding strategy to give a reasonable opinion about it. Questionnaire 2 consisted of 24 questions, with 18 questions of a competitive nature with a 5-step answering scale ranging from 'much better' to 'much worse'. The other 6 questions (Q5, Q20-24) were descriptive measurements about telephone use, music perception and music listening habits. Individual questions from both questionnaires are included in figure $1 \mathrm{a}-\mathrm{c}$ and figure $2 \mathrm{a}-\mathrm{c}$.

\section{Speech Perception Tests}

For testing of speech perception, the OLSA sentence test [1921], the Freiburg monosyllable test [22], and a vowel test [23] were used. The OLSA is a closed-set adaptive sentence test consisting in total of 40 lists with 30 sentences each. The speech level was constant at $70 \mathrm{~dB}$ SPL, and the noise level (OLSA noise) was varied in order to determine the speech-to-noise ratio (SNR) that resulted in a $50 \%$ correct speech reception threshold for each individual. The initial SNR was $10 \mathrm{~dB}$ in all subjects. One list of the OLSA tests was administered in each condition. The SNRs used in the vowel and monosyllable tests were determined from the results of a training phase preceding all speech perception tests. In this training phase, two lists of the OLSA test were administered, and the speech reception threshold resulting from the second list was used as the SNR in the vowel tests. The SNR used in the monosyllable test was then determined by adding $10 \mathrm{~dB}$ to the SNR in the vowel test. Two lists of monosyllables with 20 words each and 10 runs of the vowel test were performed in each condition. Speech perception testing was carried out in free field with both the speech and the noise presented from a loudspeaker at a distance of $1 \mathrm{~m}$ from the subject's head at $0^{\circ}$ azimuth. The order of the speech perception tests as well as of the coding strategies in all tests was randomized.

\section{Pitch Scaling}

Pitch scaling was performed using pure tones with frequencies between 110 and 1,245 $\mathrm{Hz}(110,139,156,185,220,262,311,370$, $440,523,622,740,880,1,047,1,245 \mathrm{~Hz}$ ). The tones were $500 \mathrm{~ms}$ in duration and had on- and off-ramps of $50 \mathrm{~ms}$. Each stimulus in the pitch scaling test consisted of a reference tone followed by a test tone. The reference tone had a frequency of $370 \mathrm{~Hz}$. The test tone presented one of the above-mentioned frequencies (including $370 \mathrm{~Hz}$ ). The interval between the reference tone and the test tone was $300 \mathrm{~ms}$ in duration.

Subjects were asked to indicate the magnitude of the difference in pitch between the reference tone and the test tone on an open scale, which did not provide any units or end points. Rather, each subject could establish his/her own numerical units and end points. The middle of the scale was marked with the digit 0 (zero).
Subjects were asked to allocate negative pitch differences (i.e. stimuli where the pitch of the test tone was lower than the pitch of the reference tone) left of the middle, and positive pitch differences right of the middle.

Six runs, each consisting of one complete set of test tone frequencies, were performed for the FSP and the HDCIS coding strategies. Thus, for each coding strategy, each test tone frequency was presented 6 times. The order of test tone frequencies was randomized in each run and the order of coding strategies was randomized across runs.

The tones were presented at a comfortable loudness level via headphones. The headphones used (Sennheiser HD570) were big enough to completely and tightly enclose the behind-the-ear processors. In order to eliminate the effect of loudness on pitch, all tones were balanced in loudness prior to pitch scaling. A paired comparison procedure was applied whereby the level of each test stimulus was adjusted to produce equal loudness to the reference stimulus. The resulting levels were used without roving throughout the scaling. Some of the test tone frequencies are below the lower frequency range limit used in the HDCIS strategy $(250 \mathrm{~Hz}$, see above) so that the level of these test tones needed to be increased considerably in order to produce equal loudness to the reference tone. In order to account for this, an audibility criterion was defined; if the level of the test tone of a certain frequency was larger than the level of the reference tone by more than $15 \mathrm{~dB}$, then that frequency was considered inaudible and was not included in the pitch scaling.

\section{Statistical Analysis}

Quantitative data are expressed as median or mean, with either range (minimum and maximum) or standard deviation. Qualitative data are presented as absolute and relative frequencies. Quantitative data were checked for normal distribution using the Kolmogorov-Smirnov test.

For the statistical analysis of pitch scaling test results, the open scale of each subject was transformed into a normalized scale to make the pitch scaling comparable between subjects and coding strategies. Normalization was performed by dividing each pitch judgement by the maximum absolute number used by the subject in the scaling. After normalization for each subject, mean pitch judgements and standard deviation values were calculated from the 6 judgements for each test tone frequency and coding strategy. To characterize pitch scaling as a function of coding strategy in each subject, the audible frequency range and the perceived pitch range were calculated for each coding strategy. The audible frequency range is the distance in hertz between the lowest test tone frequency and the highest test tone frequency used with a certain coding strategy in the test. Thus, the audible frequency range is the complete frequency range used in the test $(110-1,245 \mathrm{~Hz})$ minus those frequencies that were found to be

Fig. 1. a Results of questionnaire 1 at test interval 3: questions relating to music, melody, singing voice and instruments. b Results of questionnaire 1 at test interval 3: questions relating to voice quality, male/female voices and dialogue in background noise. c Results of questionnaire 1 at test interval 3: questions relating to timbre and pitch. 

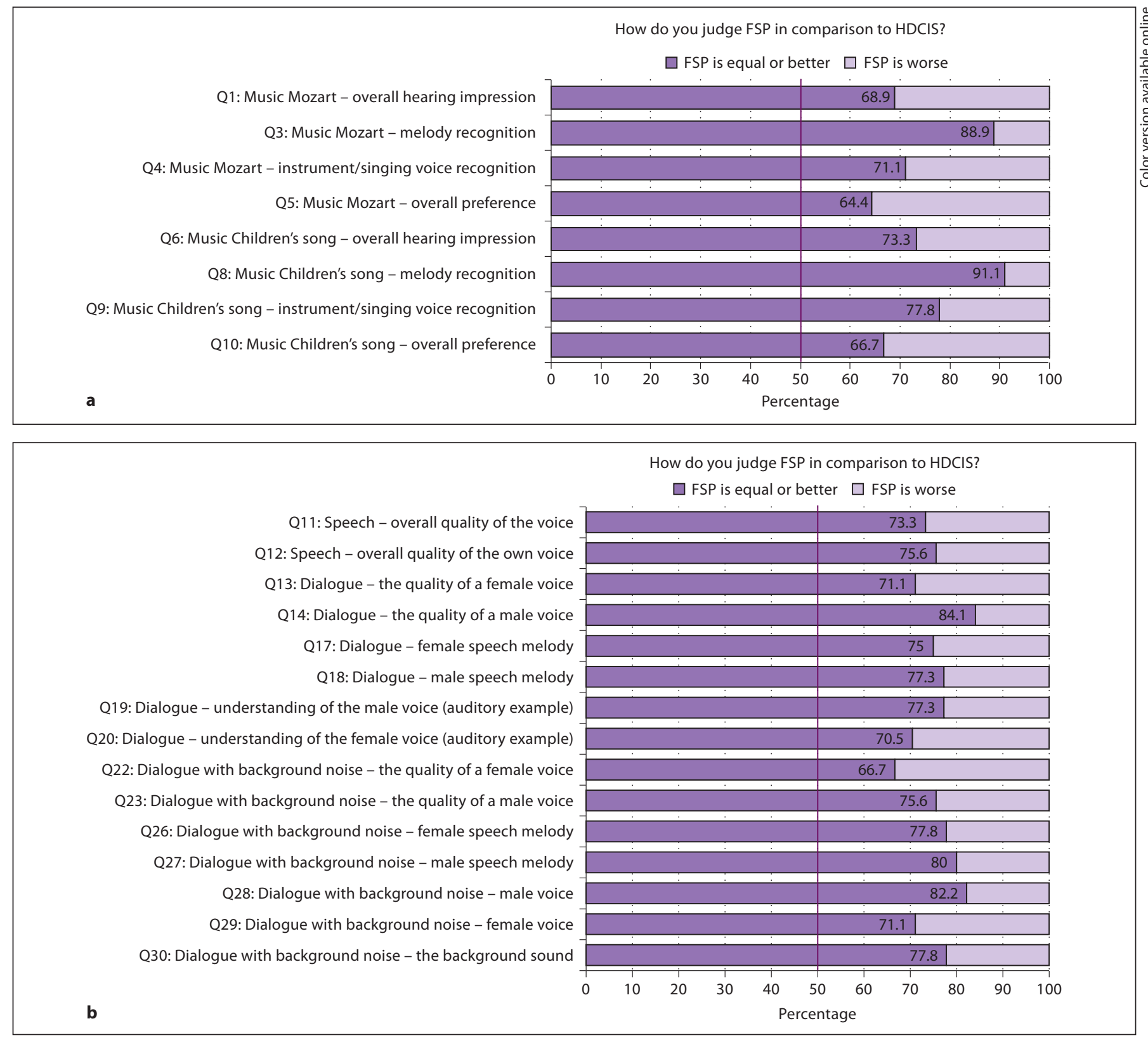

Q2: Music Mozart - timbre of sound
Q7: Music Children's song - timbre of sound
Q15: Dialogue - the female pitch Dialogue - the male pitch
Q25: Dialogue with background noise - the male pitch
C


How do you judge FSP in comparison to $\mathrm{ClS}+$ ?

$\square$ FSP is equal or better $\square$ FSP is worse

Q1: Speech understanding in quiet - female voice

Q2: Speech understanding in quiet - male voice

Q3: Speech understanding in background noise - male voice Q4: Speech understanding in background noise - female voice

Q10: Speech understanding in a group situation - male voice

Q11: Speech understanding in a group situation - female voice Q12: Speech understanding when watching TV

Q13: Speech understanding when listening to the car radio Q14: Speech understanding when listening to passengers in the car

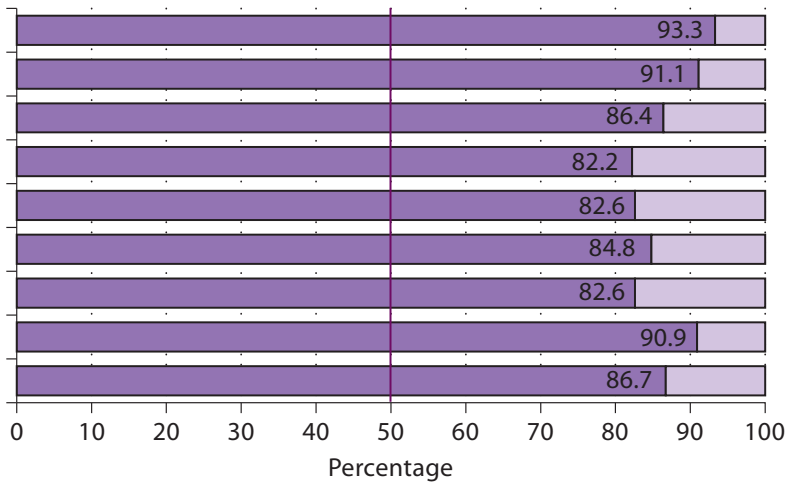

How do you judge FSP in comparison to $\mathrm{CIS}+$ ?

FSP is equal or better $\square$ FSP is worse

Q15: Listening to music - known music

Q16: Listening to music - unknown music

Q17: Melody - known music

Q18: Single musical instrument - known music

Q19: Naturalness of sounds

b

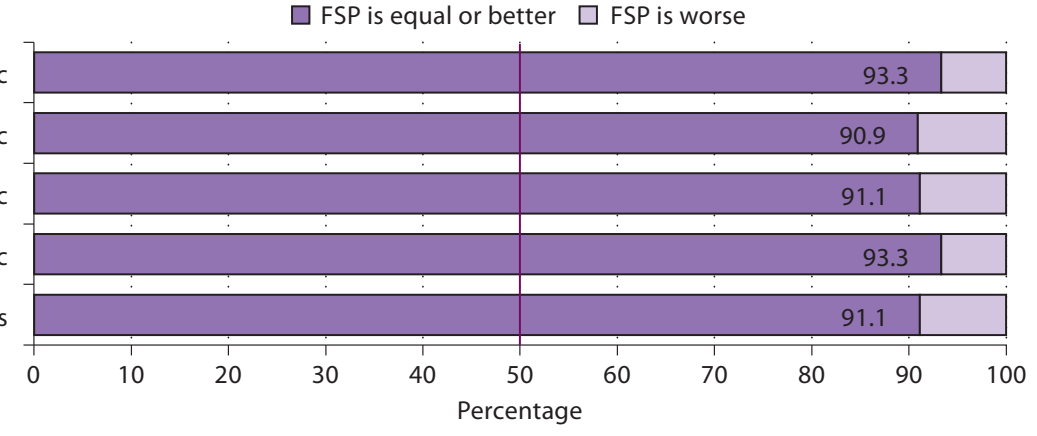

How do you judge FSP in comparison to $\mathrm{CIS}+$ ?

FSP is equal or better $\square$ FSP is worse

Q6: Speech understanding on the phone - known male voice Q7: Speech understanding on the phone - unknown male voice Q8: Speech understanding on the phone - known female voice Q9: Speech understanding on the phone - unknown female voice

c

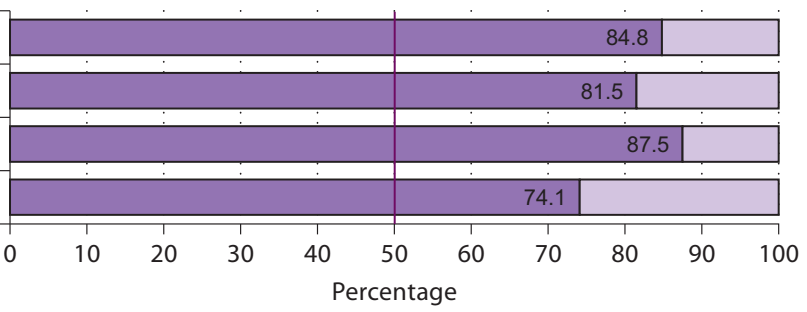

Fig. 2. a Results of questionnaire 2 at test interval 2: questions relating to speech understanding in quiet, background noise and group situations. b Results of questionnaire 2 at test interval 2: questions relating to music listening, melody and naturalness of sounds. c Results of questionnaire 2 at test interval 2: questions relating to speech understanding (male/female voices) when using the telephone. 
inaudible in the loudness balancing procedure. The perceived pitch range is the difference between the highest normalized pitch judgement and the lowest normalized pitch judgement for a certain coding strategy.

Two different statistical tests were applied to show both superiority and non-inferiority according to the study design. The methodology is described in the following two paragraphs.

Effects of coding strategy on speech perception and pitch scaling were assessed using the Sasabuchi test or the Schuirmann test [24] to show non-inferiority of FSP versus HDCIS and non-inferiority of FSP and HDCIS versus CIS+. When the KolmogorovSmirnov tests showed that data were normally distributed, the 'ratio of means' design, Sasabuchi tests and relative test bounds of $\delta=0.8$ were chosen. When the Kolmogorov-Smirnov tests indicated that data were not normally distributed, the 'difference of means log scale' design, Schuirmann tests and relative test bounds of $\delta=0.8$ were chosen.

To test superiority of FSP versus HDCIS and superiority of FSP and HDCIS versus CIS+ paired sample t tests or Wilcoxon signedrank tests were used. Whether parametric (paired $t$ test) or nonparametric (Wilcoxon signed-rank test) tests were used depended on the results of normality testing using Kolmogorov-Smirnov tests.

Effects of coding strategy on hearing quality as tested using questionnaires 1 and 2 were assessed using the binomial test to compare the frequency distributions between FSP and HDCIS.

In all statistical tests, significance was defined as $\mathrm{p}<0.05$. Equiv Test ${ }^{\mathrm{TM}} 1.0$ (Cork, Ireland, http//www.statsol.ie) was used for all non-inferiority testing. Non-inferiority testing was conducted by the Institute of Medical Statistics, Informatics, and Health Economics of the Medical University of Innsbruck. SPSS for Windows 14.0 (Chicago, Ill., USA, http//www.spss.com) was used for all other analyses.

\section{Results}

\section{Hearing Quality}

Results for questionnaires 1 and 2 are shown in figure $1 \mathrm{a}-\mathrm{c}$ and figure $2 \mathrm{a}-\mathrm{c}$. In each graph, the content of the questions is indicated on the vertical axis or in the title of the graph. Questionnaire 1 was completed at both, test interval 1 (acute fitting) and test interval 3 (after 3 months of FSP use). Data reported on are from test interval 3, as these would more realistically reflect hearing quality judgements, following some device experience.

Relative frequencies for 23 of the 24 competitive assessment questions of questionnaire 1 are shown in figure $1 \mathrm{a}$ and $\mathrm{b}$. One question was not included in figure 1a and b as $97.8 \%$ of responses were missing. With respect to music, depending on the question and the music being presented (see Methods for details), 64.4-91.1\% of the subjects rated the FSP strategy to be better than or equal to HDCIS. With respect to speech, again depending on the sound sample being used, $66.7-84.1 \%$ of the subjects rat- ed FSP to be better than or equal to HDCIS. On all questions, binomial tests showed that the proportion of subjects who have judged FSP $\geq$ HDCIS was significantly higher than (or in some cases at least equal to) the proportion of subjects who preferred HDCIS ( $p<0.05)$.

Results of the 6 questions on timbre of sound and pitch of the voice are shown in figure 1c. For these questions, the originally German answer categories are shown in English. However, please note that there is no clear translation of the German answer categories 'dunkel' ('viel dunkler' and 'dunkler') and 'hell' ('viel heller' und 'heller'). Both Langenscheidt's dictionary (eWörterbücher 4.0, Revision 14, http://www.langenscheidt.de) and the LEO dictionary (http://dict.leo.org/) translate the term 'dunkel' as 'dark', 'deep' and 'dull' and the term 'hell' as 'bright', 'light' and 'clear'. We decided to use the translation 'dark' for 'dunkel' and 'bright' for 'hell'. For these questions on timbre and pitch, it could not be defined if a brighter sound was to be preferred over a darker sound and vice versa. These questions were not of a competitive nature and were thus only included to obtain an additional idea of the quality of the different strategies. Across all questions, more subjects found FSP to sound brighter and clearer than HDCIS than there were subjects finding FSP to sound darker and 'more dull'.

After 1 month of at-home experience, subjects completed a postal questionnaire (questionnaire 2), which asked them to compare listening experiences with the OPUS 1 to those with the TEMPO+. All subjects returned this questionnaire. Some results of questionnaire 2 are shown in figure $2 \mathrm{a}-\mathrm{c}$. The open question noting different music styles subjects listened to is not depicted in these graphs as it presents no valuable information in regard to the upgrade to the new FSP coding strategy. With respect to speech understanding (fig. 2a), depending on the question, $82.2-93.3 \%$ of the subjects rated FSP $\geq$ CIS+. For questions relating to music (fig. 2b), $90.9-93.3 \%$ of the subjects rated FSP $\geq$ CIS+. Binomial tests on all competitive assessment questions showed that the proportion of subjects who judged FSP $\geq$ CIS+ was significantly higher than the proportion of subjects who preferred CIS+ $(p \leq 0.001)$.

\section{Telephone Use}

Thirteen subjects did not use the telephone; as a result, 33 (71.7\%) subjects answered questions about telephone use. As this subgroup is not a representative sample of all study participants, these questions were only analysed descriptively. On average, subjects preferred FSP over CIS+ on all telephone use questions (fig. 2c). $84.8 \%$ of the 


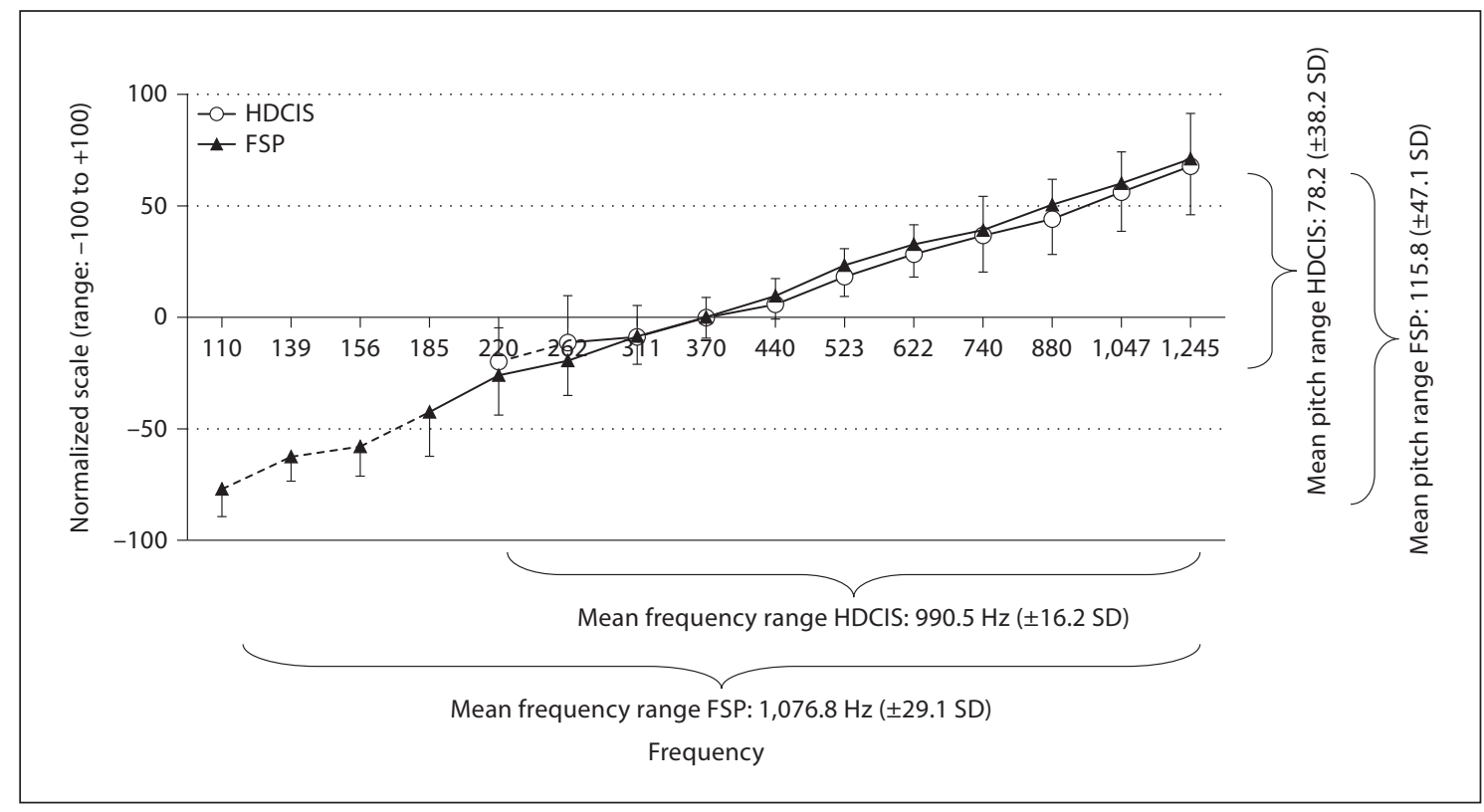

Fig. 3. Mean pitch scaling values as a function of frequency. The line with the circles shows pitch scaling for HDCIS, whereas the line with the triangles shows pitch scaling for FSP. Where the line is shown dashed, the frequency range is not applicable to all subjects. With HDCIS, 8 subjects could recognize tones down to $220 \mathrm{~Hz}$, for 37 subjects the lowest frequency was $262 \mathrm{~Hz}$. With FSP, for 7 subjects the lowest frequency was $110 \mathrm{~Hz}$ and 5 subjects could perceive the difference between tones down to $139 \mathrm{~Hz}$. For the majority of the subjects (33) 185 $\mathrm{Hz}$ was the lowest frequency. Error bars represent standard deviations of the pitch range.

subjects reported that they understood a known male communication partner as well or better with FSP in comparison to CIS+ (worse: $15.2 \%$ ). $81.5 \%$ of the subjects reported that they understood an unknown male communication partner as well or better with FSP in comparison to CIS+ (worse: $18.5 \%$ ). $87.5 \%$ of the subjects reported that they understood a known female communication partner as well or better with FSP in comparison to CIS+ (worse: $12.5 \%$ ). $74.1 \%$ of the subjects reported that they understood an unknown female communication partner better or much better with FSP in comparison to CIS+ (worse: $25.9 \%$ ).

\section{Music Listening}

$36.4 \%$ of the subjects listened to music on a daily basis, and $45.4 \%$ listened to music $1-2$ times a week. Music with the OPUS 1 sounded natural and pleasant for $39.1 \%$ of the subjects, $52.2 \%$ reported that music sounded unnatural but still pleasant; while $8.7 \%$ reported that music sounds unnatural and unpleasant to them.

$64.4 \%$ of the subjects reported that music sounds 'broad', 'full', 'resonant' and 'complete' (translation of 'voll' from http://dict.leo.org/) with FSP in comparison to
CIS+ (equal: $20 \%$, worse: $15.5 \%) .48 .9 \%$ of the subjects reported that they perceived the timbre of music to be 'brighter' with FSP in comparison to CIS+ (equal: 26.7\%, 'darker': 24.4\%).

\section{Pitch Scaling}

Again, data reported here were from test interval 3, as these more realistically reflect pitch-scaling judgements, following some device experience. Applying the audibility criterion mentioned above (see Methods section), the lowest perceivable frequency was lower using FSP (110 $\mathrm{Hz}$ ) in comparison to the lowest frequency when using HDCIS $(220 \mathrm{~Hz})$ (fig. 3). There was no difference in audibility for the highest tested frequency. In the range of perceivable frequencies, both strategies produced increasing pitch with increasing frequency. The mean audible frequency range was higher with FSP $(1,076.8 \pm 29.1 \mathrm{~Hz})$ than with HDCIS $(990.5 \pm 16.2 \mathrm{~Hz})$. A Schuirmann test confirmed non-inferiority at a significant level ( $\mathrm{p} \leq$ 0.001). A Wilcoxon signed-rank test revealed a statistically significant difference in median test results between the two groups ( $\mathrm{p} \leq 0.001$ ), indicating that subjects could hear a wider range of frequencies with the FSP strategy. 


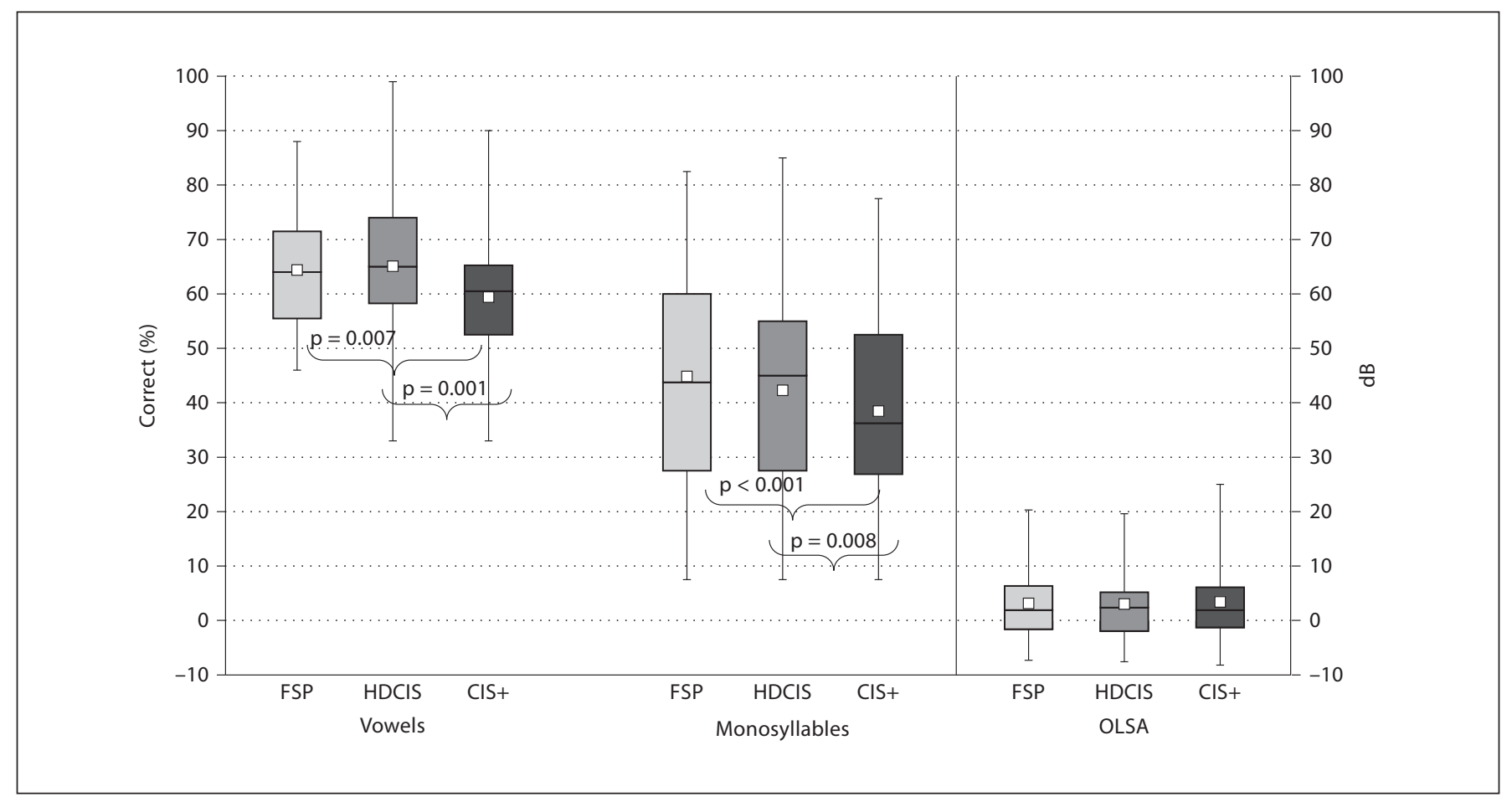

Fig. 4. Speech perception scores at test interval 3. Subjects were tested with the OLSA, Freiburg monosyllable and vowel tests using the FSP, HDCIS and CIS+ coding strategies.

The mean perceivable pitch range was higher with FSP $(115.8 \pm 47.1)$ than with HDCIS (78.2 \pm 38.2$)$. Non-inferiority of FSP was demonstrated $(\mathrm{p} \leq 0.001)$ and a paired t test showed a statistically significant difference in mean test results between the two coding strategies ( $\mathrm{p} \leq 0.001)$. This indicates that with the FSP strategy, subjects could perceive a significantly larger range of pitches than with HDCIS. Further analysis showed that this was due to differences at the lower pitch end. The mean lowest pitch score was lower with FSP $(-52.8 \pm$ 19.6) than with HDCIS (-18.6 \pm 17.3$)$. Again, non-inferiority was demonstrated ( $\mathrm{p} \leq 0.001$ ), and a Wilcoxon signed-rank test revealed a statistically significant difference in mean test results between the two strategies ( $\mathrm{p} \leq$ $0.001)$. The mean highest pitch scores were $74.0 \pm 15.2$ for FSP and $71.7 \pm 11.4$ for HDCIS. Non-inferiority was shown ( $\mathrm{p} \leq 0.001$ ), whereas a Wilcoxon signed-rank test revealed no statistically significant difference in mean test results between the two strategies $(\mathrm{p}=0.111)$.

\section{Speech Perception Testing}

Data of speech perception tests are shown in figure 4. Data were analysed for test interval 3 (3 months' device experience), as this was the defined end point of the clinical investigation, showing data with some device experience.

At test interval 3, vowel scores were similar for FSP $(64.4 \pm 10.9 \%)$ and HDCIS $(65.4 \pm 12.5 \%)[\mathrm{p} \leq 0.001$ (non-inferiority) and $\mathrm{p}=0.577$ (superiority)]. Those for FSP were significantly higher than those for CIS+ $(59.6$ $\pm 11.2 \%$ ) [p $\leq 0.001$ (non-inferiority) and $\mathrm{p}=0.007$ (paired t test - superiority)]. HDCIS vowel scores were significantly higher than CIS + scores $(\mathrm{p}=0.001)$. Monosyllable scores showed the same behaviour for FSP and HDCIS [FSP: $44.8 \pm$ 19.03\%; HDCIS: $42.3 \pm 18.8 \%$; $\mathrm{p} \leq 0.001$ (non-inferiority) and $\mathrm{p}=0.139$ (superiority)] and were also significantly higher for FSP than for CIS+ $(38.9 \pm 17.8 \%)[\mathrm{p} \leq 0.001$ (non-inferiority) and $\mathrm{p}<$ 0.001 (paired t test - superiority)]. HDCIS monosyllable scores were significantly higher than CIS + scores $(\mathrm{p}=$ 0.008). In the OLSA test, speech reception thresholds were slightly lower for FSP $(3.0 \pm 6.7 \mathrm{~dB})$ and HDCIS $(2.9 \pm 7.0 \mathrm{~dB})$ than for CIS+ $(3.4 \pm 7.7 \mathrm{~dB})$; however, none of these differences was statistically significant (fig. 4). 


\section{Discussion}

Before discussing the results, it should be noted here that although the OPUS 1 audio processor was used in the study (as this was the only audio processor available at study start), the results can be extrapolated to the OPUS 2 audio processor, as the FSP and HDCIS coding strategies are identically implemented in both processors.

Furthermore, it is to note that all tests used in this study were based on the German language which might raise the question about the applicability of our results in regard to CI patients speaking different languages, such as English. When extrapolating speech test results of German-speaking CI users to languages with radically different sound systems or phonetic structures, such as some African languages, which use an egressive airstream for the production of their typical click sounds [25], or many tonal languages (e.g. Mandarin), in which pitch changes are used to change word meaning, difficulties might occur as these linguistic devices are not found in German. In contrast, the English language, for example, has strong similarities in the consonant and vowel systems (e.g. German: 15 vowels and 3 diphthongs, and English: 12 vowels and 5 diphthongs) $[26,27]$ as well as in the use of tense and lax vowels. This strong overlap between the phonetic inventories of English and German means that listeners in both languages have access to highly similar phonetic cues. This allows the conclusion that speech data from one language can also be applied to the other language, i.e. our research results are also applicable to English-speaking CI users as well as CI users of various different languages.

\section{The Effects of Coding Strategy on Hearing Quality}

For the 5-step scale questions of questionnaire 1, categories 3-5 were considered to be 'equal to' or 'better than' - a significantly higher proportion of subjects fell within this category range (fig. la, b), indicating that the FSP strategy provides an equal or better listening percept on a subjective level in a number of listening conditions. Statistical analysis showed that, for all questions, the proportion of subjects judging FSP to be equal to, or better than, HDCIS was significantly larger than the proportion of subjects rating HDCIS better. This type of analysis is important to provide a more subjective overview of listening experiences during daily listening activities. FSP is designed to provide the listener with a better music percept due to the additional access to lower pitch and frequency, and this is indeed reflected in the outcomes of the questionnaire.
The other 6 questions (questions 2, 7, 15, 16, 24 and 25; fig. 1c) turned out to be more difficult to interpret. Mostly they were related to the perception of timbre. The choice options could be best translated as preferring either a 'darker' sound or a 'brighter' sound when relating to 'Klangfarbe' or sound colour. Although there is no direct English translation, 'Klangfarbe' might best be described as 'timbre'. Among the group analysing the data, there was much discussion (and variance) as to the meaning of the outcomes on this portion of the survey. Some expected a 'darker' sound to be richer, conveying more low-frequency information and were thus surprised by the outcome with a trend towards 'brighter' sound. Others thought that 'brighter' might translate to clearer, better listening, as this could be seen as a more positive word than 'darker'. In the end, it was agreed that the authors should not overinterpret these results, as it is difficult to assess what the subjects really intended to report, given that we have differences in opinion on this. The responses to these questions provide qualitative information regarding perception of sound and were thus not used to test any hypothesis.

All 6 questions about telephone use, music perception and music listening preferences in questionnaire 2 were analysed separately from its remaining 18 competitive questions. The relative frequency analysis on the competitive questions regarding speech and music shows that the preference for the FSP coding strategy was significantly greater than for CIS+ (fig. 2a, b). $83.9 \%$ of the subjects listened to music at least once or twice a week, if not every day, which is in contrast to research by Veekmans et al. [28] on CIS + users - where only $17 \%$ of 23 users listened to music at all. A surprisingly large number (39.1\%) reported music to sound both natural and pleasant. This contrasts Veekmans' CIS+ data, where $63 \%$ noted that music was 'pleasant noise without melody', and suggests that providing fine structure elements to the coding strategy allows users to now perceive music as more pleasant and natural, and perhaps more 'music-like'. This is supported by the fact that subjects found music to sound 'fuller' and 'brighter' with FSP. The questions regarding telephone use were descriptively analysed since not all (71.7\%) subjects reported using the telephone. A large number of subjects did use the telephone to some degree (71.7\%), which reflects the same experience in a study by Anderson et al. [29], where $71 \%$ of respondents reported using the telephone after receiving their CI. FSP may bring about better understanding, as in the current study, there were improvements on understanding of familiar (male: $84 \%$, female: $87 \%$ ) and unfamiliar speakers (male: 
$81 \%$, female: $74 \%)$. This is in comparison to the Anderson study, where $65 \%$ understood familiar speakers and $46 \%$ unfamiliar speakers well. Of those who used the telephone, most showed a preference for the FSP coding strategy (fig. 2c).

All in all, the results of the questionnaires 1 and 2 indicate that most subjects found FSP to perform equal to, or better than, CIS + with a large variety of sound signals and in a large variety of environments. In no question did FSP perform worse than HDCIS. The results regarding music are of particular interest since FSP is designed to provide better frequency information in the low frequencies. These results suggest that in contrast to earlier assessments of music appreciation with a CI [30, 31], FSP coding, which provides more information on pitch frequency and possibly - depending on the sound - also on the first few harmonics, may enhance the music listening experience. This is verified by the large number of subjects who reported that even though music might sound unnatural (which can be expected), it still sounds pleasant - suggesting access to the fine structure of sound, over and above the envelope of sound, has somewhat improved the music listening experience. In all, $91 \%$ of subjects reported that after using FSP, music is now pleasant to listen to with their CI, exceeding by far the numbers reached with CIS, ACE and SPEAK as reported in a study by Brockmeier et al. [32]. More recent studies confirm our results of an improved music perception. Arnoldner et al. [14] investigated speech and music perception in 14 postlingually deafened adults und used the MED-EL MUSIC test to assess specific musical skills and subjective judgements. Results showed that subjects performed better with the OPUS processor using FSP compared to the TEMPO+ using CIS in 2 out of 3 tests (rhythm and number of instruments). Similarly, Lorens and his research group [33] tested 60 children using a visual analogue scale to assess user satisfaction regarding music stimuli. In the visual analogue scale at interval II (3-4 months of HDCIS experience only, none with FSP) subjects rated FSP better than CIS+ by $27.1 \%$, HDCIS better than CIS+ by $31.5 \%$ and no significant difference between FSP and HDCIS. At interval III (3-4 months of FSP experience only), subjects rated FSP better than CIS+ by $32.4 \%$, HDCIS better than CIS+ by $22.3 \%$ and again no significant differences between HDCIS and FSP. Thus, we can conclude that our outcomes in adults also concur with findings in children.

\section{The Effects of Coding Strategy on Pitch Scaling}

The results from the questionnaires are complemented by the results of the pitch scaling experiments. With the FSP strategy, pitch scaling showed a significantly greater access to lower frequencies (lowest audible frequency for FSP: $110 \mathrm{~Hz}$ vs. for HDCIS: $220 \mathrm{~Hz}$ ) and a significantly lower pitch percept (lowest perceived pitch for FSP: -58.1 vs. -12.8 for HDCIS) (fig. 3). This difference of pitch scaling results between FSP and HDCIS/CIS+ coding strategies cannot only be attributed to the fine structure processing but also to the lower cut-off frequency for FSP at $100 \mathrm{~Hz}$ compared to $250 \mathrm{~Hz}$ for CIS+ and HDCIS strategies. As expected, no significant difference in audible frequency or perceived pitch was found at the upper end of the tested frequency range. Here, no technical difference exists between FSP and HDCIS, i.e. both strategies provide envelope information only, so that no difference was to be expected.

Thus, the results show that the FSP strategy provides the listener with better pure-tone hearing for frequencies lower than $220 \mathrm{~Hz}$ indicating that the FSP strategy meets its principal design goal, i.e. better frequency coding in the lower frequencies. This obviously gives the listener greater access to pitch which could provide better access to the fundamental frequency (F0). Better F0 could improve speech perception in a variety of listening conditions and improve access to music via melody recognition, which is in line with the results of the questionnaires discussed above. Interestingly, however, an experiment by Krenmayr et al. [34], in which they compared two different fine structure strategies, demonstrated that the pitch of a stimulus does not only depend on its F0 but also on the presentation of acoustic properties in stimulation patterns. They furthermore concluded that fine structure enables CI users to perceive lower pitches than purely enveloped-based coding strategies such as CIS/CIS+.

\section{The Effects of Coding Strategy on Speech Perception}

Results showed that subjects performed similarly with FSP and HDCIS but significantly better with FSP and HDCIS than with CIS+ on vowels and monosyllables (tables 2 and 3). Improved vowel and monosyllabic word scores support the claim suggested by results of the questionnaires (see above) that FSP may indeed provide better everyday listening outcomes for users than the CIS+ strategy. Our improved speech perception results in quiet are in line with multiple other studies showing a benefit of FSP in speech understanding in children and adults $[14,33,35-37]$. However, whereas sentence perception in noise was not significantly different with FSP and HDCIS 
Table 2. Results for speech understanding scores (vowels, monosyllables, OLSA adaptive sentences in noise) at 3-month test intervals (test interval 3)

\begin{tabular}{|c|c|c|c|c|c|c|c|c|c|}
\hline & \multicolumn{9}{|c|}{ Speech understanding at test interval 3} \\
\hline & \multicolumn{3}{|l|}{ vowels } & \multicolumn{3}{|c|}{ monosyllables } & \multicolumn{3}{|l|}{ OLSA } \\
\hline Mean & 64.40 & 65.38 & 59.62 & 45.00 & 42.78 & 38.94 & 2.95 & 2.86 & 3.26 \\
\hline Median & 64.00 & 65.00 & 61.00 & 45.00 & 45.00 & 37.50 & 1.50 & 2.10 & 1.60 \\
\hline Maximum & 88.00 & 99.00 & 90.00 & 82.50 & 85.00 & 77.50 & 20.30 & 19.60 & 25.00 \\
\hline
\end{tabular}

Table 3. Results for speech understanding scores (vowels, monosyllables, OLSA adaptive sentences in noise) at test interval 3: $\mathrm{p}$ values

\begin{tabular}{llcl}
\hline Test & $\begin{array}{l}\text { Non-inferiority and } \\
\text { superiority of FSP }\end{array}$ & $\begin{array}{c}\text { FSP vs. } \\
\text { HDCIS }\end{array}$ & $\begin{array}{l}\text { FSP vs. } \\
\text { CIS+ }\end{array}$ \\
\hline \multirow{2}{*}{ Vowels } & Non-inferiority of FSP & $\leq 0.001^{*}$ & $\leq 0.001^{*}$ \\
& Superiority of FSP & 0.577 & $0.007^{*}$ \\
\hline Monosyllables & Non-inferiority of FSP & $\leq 0.001^{*}$ & $\leq 0.001^{*}$ \\
& Superiority of FSP & 0.139 & $<0.001^{*}$ \\
\hline OLSA & Non-inferiority of FSP & $\leq 0.001^{*}$ & $\leq 0.001^{*}$ \\
& Superiority of FSP & 0.734 & 0.330 \\
\hline
\end{tabular}

${ }^{*} \mathrm{p}<0.05$ : results indicating statistical significance.

from CIS+ in our study, study outcomes by Vermeire et al. [37] as well as other research groups $[14,33,36]$ demonstrated statistically significant improvement of speech understanding in noise with FSP compared to the CIS or CIS+ coding strategy. It is to note, however, that the follow-up time in both Vermeire's [37] and Riss' [36] studies was 12 months, which indicates that subjects might need more time to get fully accustomed to the FSP coding strategy and to learn to use the cues that are available with FSP to greater benefit in speech understanding in noise.

Interestingly, the HDCIS speech perception test results in our study were quite similar to those of the FSP coding strategy. When considering the results from questionnaire 1 (comparing FSP and HDCIS, with a significant preference for FSP) and questionnaire 2 (comparing FSP and CIS+, with a significant preference for FSP), this comes as a surprise considering that CIS+ and HDCIS are essentially the same coding strategy. Perhaps speech perception measures are not sensitive enough to the changes that come with increased lower frequencies. This could suggest that there are subtle changes that affect everyday listening that cannot be measured in a laboratory setting and suggests that the development of improved test methods is important. In addition, these results may again indicate that subjects need a longer follow-up time with FSP in order to get used to this coding strategy and to show greater differences between FSP and previous coding strategies.

\section{Conclusions}

Results from this clinical study demonstrate that users of FSP as implemented in the OPUS processor performed as well or even better when compared to their scores with CIS+ or HDCIS. These results also show that CIS users should have no decrement in performance when they transition to the FSP coding strategy. Subjects performed significantly better on vowel and monosyllabic word perception tests with the OPUS processor than with the TEMPO+. The study demonstrated a significantly lower pitch perception and significant user preference for FSP in questionnaire 2. Coupled with improved rating of music appreciation as well as finding regular music listening habits among subjects, these results not only suggest the superiority of the new FSP coding strategy, but also indicate that the addition of the time code in FSP does indeed offer an important new direction in overcoming the limitations of traditional CI coding strategies. 


\section{Acknowledgements}

The authors would like to acknowledge the following clinics that provided subjects for this study: Klinik und Poliklinik für Hals-, Nasen- und Ohrenkrankheiten, Universitätsklinikum Würzburg, Würzburg; Hals-Nasen-Ohren Klinik, Klinikum rechts der Isar der TU München, Munich; HNO- und Poliklinik, Klinikum Grosshadern; Hals- Nasen-Ohrenklinik der RuhrUniversität Bochum, St Elisabeth Hospital, Bochum; Zentrum der Hals-Nasen-Ohrenheilkunde der Universität, Klinikum JW. Goethe-Universität, Frankfurt; Katholisches Klinikum Koblenz Marienhat, Koblenz; Klink- und Poliklinik für HNO-Heilkunde, Universitätsklinikum Carl Gustav Carus, Dresden; Klinik für
Hals-Nasen-Ohrenheilkunde, HELIOS Klinikum Erfurt GmbH, Erfurt; Poliklinik: Plastische Operationen, Universitätsklinik für Hals-, Nasen- und Ohrenheilkunde, Essen; Klinik und Poliklinik für Hals-, Nasen- und Ohrenheilkunde, Universitätsklinikum Hamburg-Eppendorf, Hamburg; Hals-, Nasen-, Ohrenheilkunde, Medizinische Hochschule Hannover, Hannover; Institut für Phoniatrie und Pädaudiologie, Universitätsklinikum Jena, Jena; Klinik für Hals- Nasen- und Ohrenheilkunde, Medizinische Fakultät, Otto-von-Guericke-Universität Magdeburg, Magdeburg; HNO Klinik Otto Körner, Universtität Rostock, Rostock; Universitäts-HNO-Klinik, Tübingen; Hals-, Nasen-, Ohrenheilkunde, Universitätsklinikum Ulm, Ulm. This clinical investigation was sponsored by MED-EL GmbH.

\section{References}

1 Hilbert D: Grundzüge einer allgemeinen Theorie der linearen Integralgleichungen. Leipzig, Teubner, 1912.

2 Pickles JO: An Introduction to the Physiology of Hearing, ed 3. Bingley, Emerald Group Publishing Ltd, 2008.

3 Smith ZM, Delgutte B, Oxenham AJ: Chimaeric sounds reveal dichotomies in auditory perception. Nature 2002;416:87-90.

4 Eddington DK: Future Directions: Questions Deserving Attention. Conference on Implantable Auditory Prostheses, Asilomar Conference Center, Pacific Grove, August 1999.

5 Wilson BS: Strategies for representing speech information with cochlear implants; in Niparko JK, Kirk KI, Mellon NK, Robbins AM, Tucci DL, Wilson BS (eds): Cochlear Implants: Principles and Practices. Philadelphia, Lippincott Williams \& Wilkins, 2000, pp 129-170.

6 Zeng FG, Nie K, Stickney G, Kong YY: Auditory perception with slowly-varying amplitude and frequency modulations; in Pressnitzer D, de Cheveigne A, McAdams S, and Collet L (eds): Auditory Signal Processing: Physiology, Psychoacoustics, and Models. New York, Springer, 2004, pp 282-290.

7 Wong A, Wong L: Tone perception of Cantonese speaking prelingually hearing impaired children with cochlear implants. Otolaryngol Head Neck Surg 2004;130:751-758.

8 Fu QJ, Chinchilla S, Galvin JJ: The role of spectral and temporal cues in voice gender discrimination by normal-hearing listeners and cochlear implant users. J Assoc Res Otolaryngol 2004;5:253-260.

$\checkmark 9$ McDermott H: Music perception with cochlear implants: a review. Trends Amplif 2004;8:49-82.

10 Von Ilberg C, Kiefer J, Tillein J, Pfennigdorf T, Hartmann R, Stürzebecher E, Klinke R: Electric-acoustic stimulation of the auditory system. ORL 1999;61:334-340.

11 Kiefer J, Pok S, Adunka O, Sturzebecher E, Baumgartner W, Schmidt M, Tillein J, Ye Q,
Gstoettner W: Combined electric and acoustic stimulation of the auditory system: results of a clinical study. Audiol Neurotol 2005;10: 134-144.

12 Helms J, Müller J, Schön F, Winkler F, Shehata-Dieler W, Kastenbauer E, Baumann U, Rasp G, Schorn K, Ebetaer B, Baumgartner W, Hamzavi S, Gstöttner W, Westhofen M, Döring W, Dujardin H, Albegger K, Mair A, Zenner H, Haferkamp C, Schmitz-Salue C, Arold R, Sesterhenn G, Jahnke V, Wagner H, Gräbel S, Bockmühl U, Häusler R, Vischer M, Kompis M, Hildmann H, Radü H, Stark T, Engel A, Hildmann A, Streitberger C, Hüttenbrink K, Müller-Aschoff E, Hofmann G, Seeling K, Hloucal U, von Ilberg C, Kiefer J, Pfennigdorf T, Gall V, Breitfuss A, Stelzig Y, Begall K, Hey M, Vorwerk W, Thumfart W, Gunkel A, Zorowka P, Stephan K, Gammert C, Mathis A, DeMin N, Freigang B, Ziese M, Stützel A, von Specht H, Arnold W, Brockmeier S, Ebenhoch H, Steinhoff A, Zierhofer C, Zwicknagl M, Stöbich B: Comparison of the TEMPO+ ear-level speech processor and the CIS PRO+ body-worn processor in adult MED-EL cochlear implant users. ORL 2001; 63:31-40.

13 Zierhofer C: Electrical nerve stimulation based on channel specific sampling sequences. US patent No 6,594,525 B1, July 15, 2003.

14 Arnoldner C, Riss D, Brunner M, Durisin M, Baumgartner W, Hamzavi J: Speech and music perception with the new fine structure speech coding strategy: preliminary results. Acta Otolaryngol 2007;127:1298-1303.

15 Looi V, Winter P, Anderson I, Sucher C: A music quality rating test battery for cochlear implant users to compare the FSP and HDCIS strategies for music appreciation. Int J Audiol 2011;50:503-518.

16 Wilson BS, Lawson DT, Zerbi M, Finley CC: Virtual channels interleaved sampling (VCIS) processor. First Quarterly Progress Report, NIH Contract N01-DC-2-2401, Neural Prosthesis Program, National Institutes of Health, Bethesda, 1992.
17 Wilson BS, Zerbi M, Lawson DT: Identification of virtual channels conditions on the basis of pitch. Third Quarterly Progress Report, NIH Contract N01-DC-2-2401, Neural Prosthesis Program, National Institutes of Health, Bethesda, 1993.

18 Nobbe A, Schleich P, Zierhofer C, Nopp P: Frequency discrimination with sequential or simultaneous stimulation in MED-EL cochlear implants. Acta Otolaryngol 2007;127: 1266-1272.

19 Wagener K, Kollmeier B, Kühnel V: Entwicklung und Evaluation eines Satztests in deutscher Sprache. 1. Design des Oldenburger Satztests. Z Audiol 1999;38:4-15.

20 Wagener K, Brand T, Kollmeier B: Entwicklung und Evaluation eines Satztests in deutscher Sprache. 2. Optimierung des Oldenburger Satztests. Z Audiol 1999;38:44-56.

21 Wagener K, Brand T, Kollmeier B: Entwicklung und Evaluation eines Satztests in deutscher Sprache. 3. Evaluation des Oldenburger Satztests. Z Audiol 1999;38:86-95.

22 Hahlbrock KH: Wörter für Gehörprüfung mit Sprache; in Hahlbrock KH: Sprachaudiometrie, ed 2. Thieme, Stuttgart, 1970.

23 Helms J, Müller J, Schön F, Moser L, Arnold W, Janssen T, Ramsden R, von Ilberg C, Kiefer J, Pfennigdorf T, Gstöttner W, Baumgartner W, Ehrenberger K, Skarzynski H, Ribari O, Thumfart W, Stephan K, Mann W, Heinemann M, Zorowka P, Lippert KL, Zenner HP, Bohndorf $M$, Hüttenbrink $K$, MüllerAschoff E, Hofmann G, Freigang B, Begall K, Ziese M, Forgbert O, Häusler R, Vischer M, Schlatter T, Schlöndorff G, Korves B, Döring H, Gerhardt HJ, Wagner H, Schorn K, Schilling V, Baumann U, Kastenbauer E, Albegger K, Mair A, Gammert C, Mathis A, Streitberger C, Hochmair-Desoyer I: Evaluation of performance with the COMBI40 cochlear implant in adults: a multicentric clinical study. ORL 1997;59:23-35.

-24 Berger RL, Hsu JC: Bioequivalence trials, in tersection-union tests and confidence sets. Stat Sci 1996;11:283-319. 
25 Crystal D: The Cambridge Encyclopedia of Language. Cambridge, Cambridge University Press, 1987.

26 Wells JC: German. http://www.phon.ucl. ac.uk/home/sampa/german.htm. Updated March 18, 2006 (accessed November 11, 2011).

27 Wells JC: English. http://www.phon.ucl. ac.uk/home/sampa/english.htm. Updated March 18, 2006 (accessed November 11, 2011).

28 Veekmans K, Ressel L, Mueller J, Vischer M, Brockmeier SJ: Comparison of music perception in bilateral and unilateral cochlear implant users and normal-hearing subjects. Audiol Neurootol 2009;14:315-326.

29 Anderson I, Baumgartner WD, Böheim K, Nahler A, Arnolder C, D’Haese P: Telephone use: what benefit do people with a cochlear implant receive? Int J Audiol 2006;45:446453.
30 Tyler RS, Gfeller K, Mehr MA: A preliminary investigation comparing one and eight channels at slow rates on music appraisal in adults with cochlear implants. Cochlear Implants Int 2000;1:82-87.

31 Brockmeier SJ, Nopp P, Vischer M Baumgartner W, Stark T, Schön F, Müller J, Braunschweig T, Busch R, Getto M, Arnold W, Allum DJ: Correlation of speech and music perception in postlingually deaf Combi 40/40+ users; in Kubo T, Takahashi Y, Iwaki $\mathrm{T}$ (eds): Cochlear Implants: An Update: The Hague, Kugler Publications, 2002, pp 459464.

32 Brockmeier SJ, Grasmeder M, Passow S, Mawmann D, Vischer $M$, Jappel A, Baumgartner W, Stark T, Müller J, Brill S, Steffens T, Strutz J, Kiefer J, Baumann U, Arnold W: Comparison of musical activities of cochlear implant users with difference speech-coding strategies. Ear Hear 2007; 28(2 suppl):49S-51S.

33 Lorens A, Zgoda M, Obrycka A, Skarzynski $\mathrm{H}$ : Fine structure processing improves speech perception as well as objective and subjective benefits in pediatric MED-EL COMBI 40+ users. Int J Pediatr Otorhinolaryngol 2010;74:1372-1378.
34 Krenmayr A, Visser D, Schatzer R, Zierhofer C: The effects of fine structure stimulation on pitch pitch perception with cochlear implants. Cochlear Implants Int 2011;12:70-72.

- 35 Riss D, Hamzavi JS, Katzinger M, Baumgartner WD, Kaider A, Gstoettner W, Arnoldner C: Effects of fine structure and extended low frequencies in pediatric cochlear implant recipients. Int $\mathrm{J}$ Pediatr Otorhinolaryngol 2011;75:573-578.

36 Riss D, Arnoldner C, Reiss S, Baumgartner WD, Hamzavi JS: 1-year results using the Opus speech processor with the fine structure speech coding strategy. Acta Otolaryngol 2009;129:988-991.

37 Vermeire K, Kleine-Punte A, Van de Heyning $\mathrm{P}$ : Better speech recognition in noise with the fine structure processing coding strategy. ORL J Otorhinolaryngol Relat Spec 2010; 72:305-311. 